\title{
Do pacifiers interfere with breastfeeding success?
}

The use of pacifiers is an
ancient practice, but often
becomes a point of debate
between parents and
professionals. The research of
Dr Alejandro Gustavo Jenik,
a paediatrician at Hospital
Italiano de Buenos Aires,
Argentina, aims to explore
the relationships between
pacifier use and breastfeeding.
His current research is
investigating the impact of
early introduction of pacifiers
on breastfeeding prevalence
and duration.

udden unexpected infant death (SUID), including sudden infant leading cause of death in the developed world for infants aged one month to one year of age. Recent SIDS rates vary across high-income countries (2012-2014), ranging from 0.05 deaths per 1,000 live biths in the US SUIB por l,000 live sudden, unexpected death of infats when subsequent investigations fail to demonstrate a definite cause of death. Other causes of SUID must be considered and excluded before a diagnosis of SIDS can be established.

Dr Alejandro Gustavo Jenik is a paediatrician based in the Neonata Department at the Hospital Italiano in Buenos Aires. His research into SIDS has included investigating pacifier use in relation to breastfeeding.

Dr Jenik works alongside Dr Nestor Vain, who contributed to the design Weiss MSc, PhD, CChem. FPSC MRSH (now deceased) was Dr Jenik's mento in regards pacifiers. DrWeiss is leading authorities on infant especially in relation to the clinical effects on babies and young children. Dr Weiss's interest also included the possible effects of chemicals used in the production of baby products. All the colleagues belonging to the Department of Neonatology of the Hospital Italiano had a collaborative thude thands study, which allowed

PACIFIERS AND

BREASTFEEDING SUCCESS The real debate over pacifiers has the establishment and duration of breastfeeding. Lactation consultants believe that use of pacifiers interferes with breastfeeding and significantly decreases duration of lactation, but Dr Jenik did not believe this to be the case. Evidence to the contrary includes a randomised controlled trial developed in Argentina. The objective was to evaluate whether the recommendation to offer a pacifier once lactation was well established reduced the prevalence or duration of lactation. The population included 1023 mothers highly motivated birth weight by 15 days. Moth regained randomly assigned to one of two groups offering a pacifier and not a offering pacifier. The offering group received package containing six silicone pacifiers, supplied by MAM Babyartikel Gesmbh (www.mambay.com/professionals) and not sold in Argentina. The study demonstrated that when mothers are determined to breastfeed for more than three months and they are successfully breastfeeding at two weeks, the advice to use or not to use a pacifier does not affect breastfeeding

BABY FRIENDLY

HOSPITAL INITIATIVE

The Ten Steps to Successfru Breastteeding' declaration
was established following a meeting

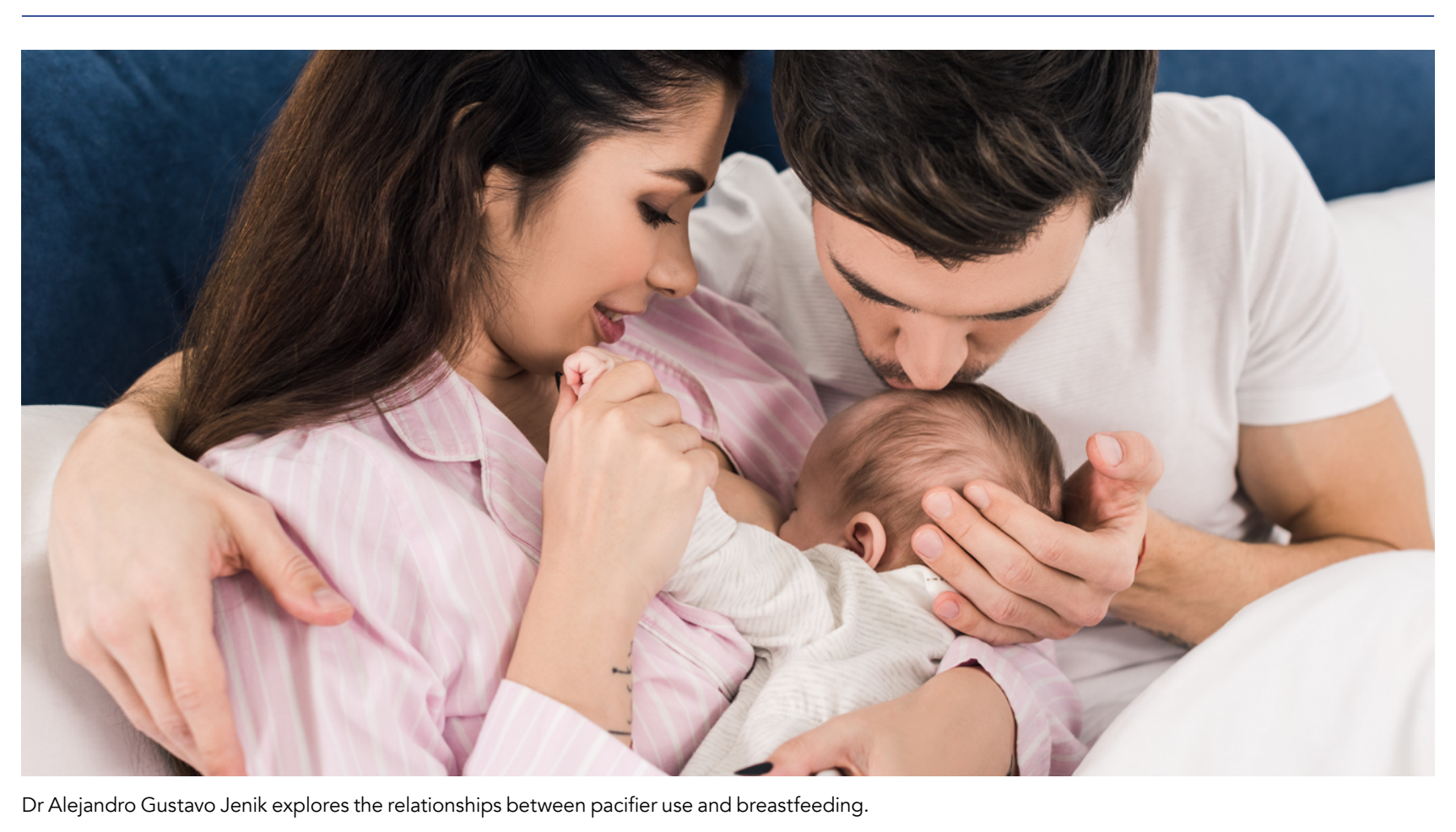

between international health

organisations to help governments a joint WHO/UNICEF Statement in 1989 . Step 9 of the statement recommends tota avoidance of artificial teats or pacifiers for breastfeeding infants. The declaration was mandatory at hospitals certified as 'Baby Friendly Hospitals' by UNICEF. When the 'Ten Steps' were developed in 1989, there was no epidemiological evidence showing that pacifier use was detrimental
to breasffeeding. The to breastfeeding. Th first observational study which concluder that pacifiers are causally associated with weaning was published in The
Lancet in 1993. The investigation

regarding pacifier and breastfeeding continued. Whilst many observational studies have demonstrated a negative association between pacifier use and breastfeeding duration, observational studies cannot be used to determine whether the pacifier is the single cause of breastreeding cessation or is simply a marker of breastfeeding difficulties.

Results from four randomised controlled trials revealed no difference in breastfeeding outcomes at different types of pacifier interventions. The Jenik Study

study from Canada $(n=281)$ were included in the Cochrane review.

The topic of step 9 has been updated (2018) according to evidence-based medicine guidelines: risks of feeding bottles, teats and pacifiers, a phrase which neither of pacifiers alongside breastfeeding

Sudden unexpected infant death, including sudden infant death syndrome, is the leading cause of death study showed that the developed world for infants aged of pacifiers was one month to one year of age. $\quad \begin{aligned} & \text { manily confined to } \\ & \text { those who shared }\end{aligned}$ This modification is a paradigm shift that allows families to make informed decisions on the use or avoidance of pacifiers.

PACIFIERS AND SUDDEN INFANT DEATH SYNDROME A landmark study carried out in 1993 firs showed the connection between SIDS and pacifiers, specifically, the reduced risk of SIDS if a pacifier had been used This was supported by eleven further case-control studies (which compare a group which have not received the reatment). These studies showed that risk of SIDS was reduced by approximately meta-analyses, which analyse the results of multiple studies, also reached

The reduced risk of SIDS appeared to be greater when pacifiers were used in fants with adverse sleep conditions, their side, as well as those who co-sleep siblings. Interestingly, the use of a pacifier at the onset of sleep appeared protective, even if the pacifier falls out of the mouth after the infant falls asleep.

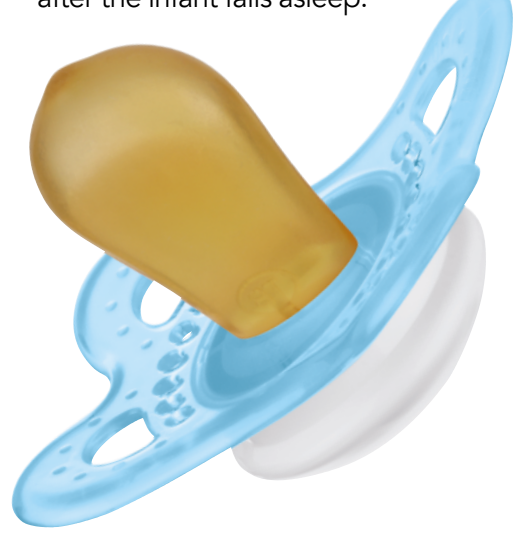




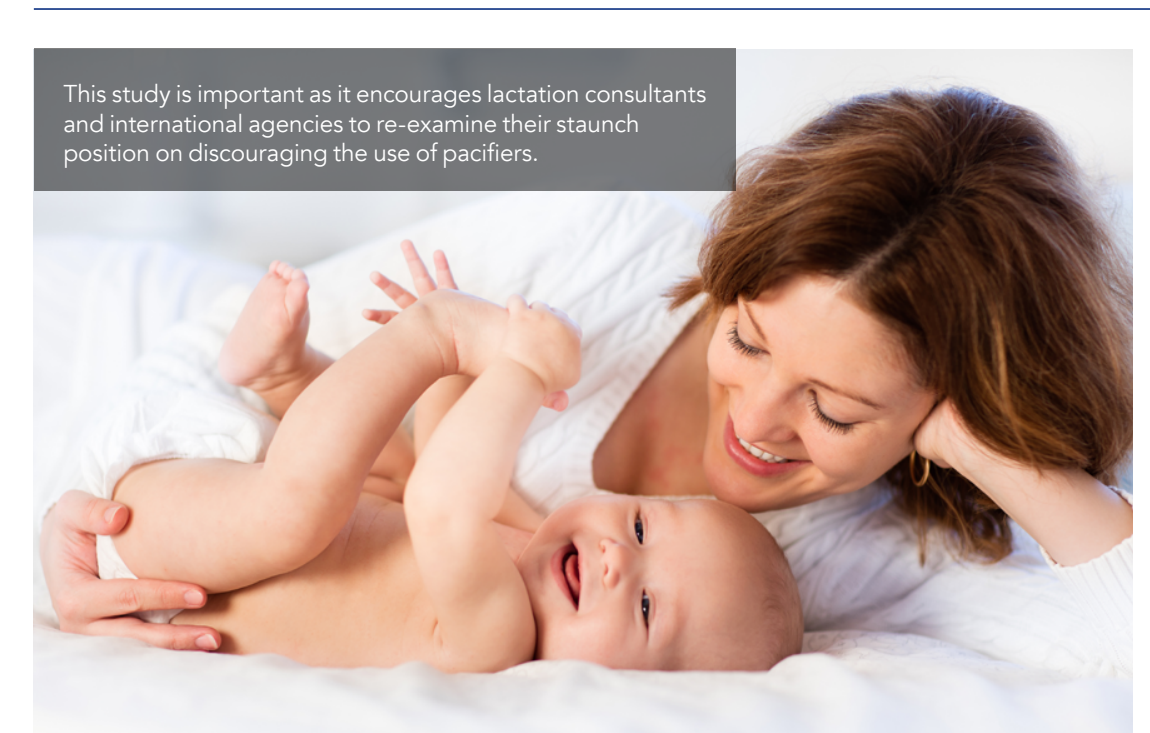

The way in which a pacifier can reduce the risk of SIDS is still unclear but there
are numerous mechanisms proposed were being exclusively breastfed Furthermore, the recommendation to offer a pacifier did not produce

include improved autonomic control a significant decrease in the frequency

Mothers who are motivated to breastfeed their infants should be allowed to make their own decisions regarding pacifier use.

over into the prone position. They also prevalence of breastfeeding at any age, help to maintain upper airway patency increase blood pressure during sleep and induce a forward movement of the jaw which may facilitate breathing

\section{CONCLUSIONS OF THE JENIK} The restls of Dr Jenik's study showed in the pacier group and $86.2 \%$ of

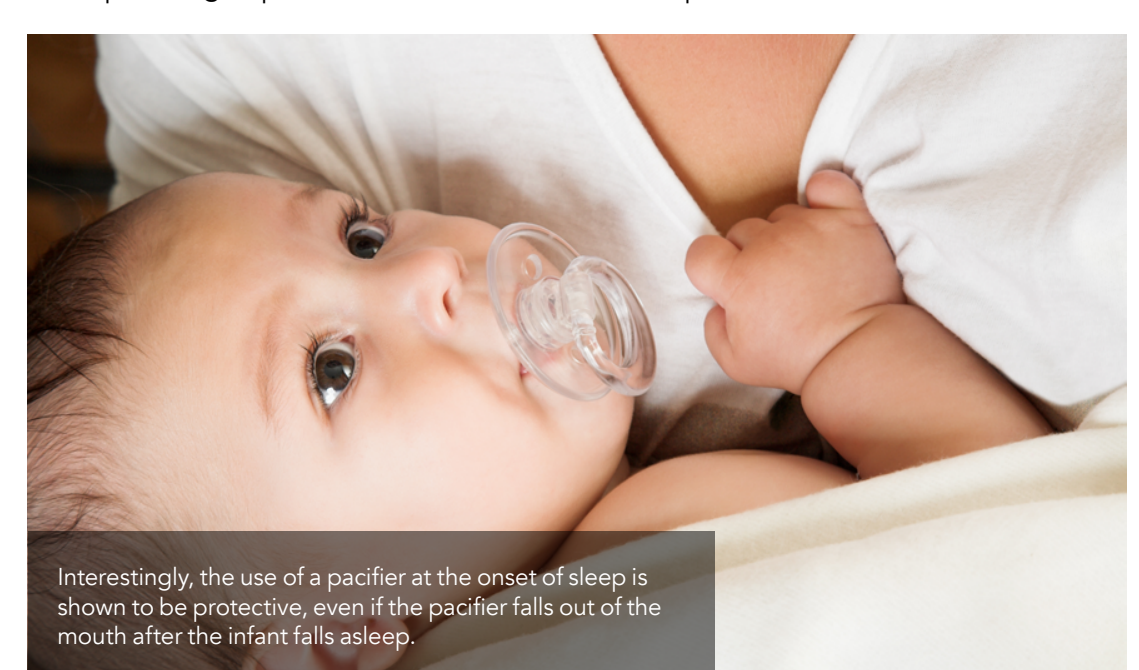

to Reduce the Risk of Sudden Infant Death Syndrome" document.

This study is therefore important as it encourages lactation consultants and their staunch position on discouraging the use of pacifiers, due to adverse effect on breastfeeding. It paves the way for physicians, nurses and healthcare staff o continue supporting breasffeeding without needing to avoid the use of pacifiers; an intervention that has been clearly demonstrated to reduce the ris of SIDS.

THE MISSING ANSWERS: WHEN IS IT SAFE TO INTRODUCE PACIFIER TO THE BREASTFED BABY Evidence is particularly lacking to guide advice regarding pacifer use in the The conclusions of a Cochrane study suggest that the introduction of pacifiers at 14 days or later has no detrimental effect on breastfeeding, but there is less evidence exploring the impact of pacifie use from birth. Studies on the risks and benefits of pacifiers are hampered by the issue of reverse causality. For example, does pacifier use have an adverse effect on breastfeeding, or it is a marker for breast feeding or weaning difficulties? It is this evidence gap that Dr Jenik hopes to fill.

More research is still needed to define whether pacifier use has any impact on to answer this question, Dr Jonik and colleagues plan to study to assess the impartake another a pacifier on breasteeding during these first days of life.

HOW ARE PACIFIERS PROTECTIVE IN THE SLEEP ENVIRONMENT? One recent study suggests that pacifiers lower the auditory arousal threshold. They may provide a mechanical barrier to rolling over into the prone position. Sucking on a pacifier keeps the tongue forward maintaining upper airway patency. An infant who is soothed by pacifier may not move as often during sleep, thus limiting the chance of becoming covered by blankets. It has also been suggested that pacifier use could and incresse carbon dioxide retention

\section{Behind the Research}

Dr Alejandro Jenik

E: alejandro.jenik@hospitalitaliano.org.ar W: www.sids.net.ar

Research Objectives

A neonatal specialist, the research of Alejandro Gustavo Jenik focuses on various aspects of child care: breastfeeding pacifiers, safe sleep, preterm feeding, bedsharing, and early skin-to-skin contact.

\section{Detail}

Alejandro Gustavo Jenik

Olivos. Provincia de Buenos Aires. Argentina

Bio

Dr Alejandro Jenik is a well-known neonatology

specialist at the Hospital Italiano de Buenos Aires. He holds a leading role in SIDS research in Latin America. He has led workshops, conferences and worked on this topic with specialists throughout the world. He was the
vice president of the International Conference on SIDS that took place in Montevideo in 2016.

Funding

This study was funded by International

Children Medical Research Asso

Switzerland (https://www.icmrs.org)

\section{Principal authors}

Jenik Alejandro, Vain Nestor, Gorestein Adriana, Jacobi Noemi and the Pacifier and Breastfeeding Trial group.

\section{Collaborators}

- Guillermo Caroli, Gusatvo Izbizky, Edgardo Szyld, Carlos Fustinana (deceased) for thoughtful advice - Richard Schanler, M. Jeffrey Maisels, Waldemar Carlo, and Gonzalo Mariani for reviewing the manuscript - David Mage (deceased) and Adele Engelberts for ther comments and support

- Daniel Wojdyla for statistical recommendations - Veronica Marino for secretarial assistance who participated in this trial
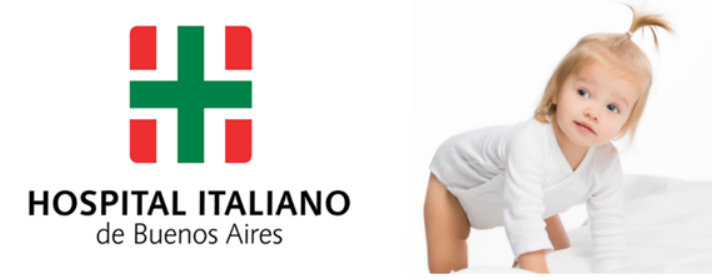

\section{References}

Jenik, A.G., Vain, N.E, Gorstein, A.N. \& Jacobi, N.E. (2009). Does the Recommendation to Use a Pacifier Influence the 155(3):350-4.e1

Jaafar SH, Jahanfar S, Angolkar M, et al. Pacifier use versus no pacifier use in breastfeeding term infants for increasing duration of breastfeeding. Cochrane Database Syst Rev. 2011;(3):CD007202

\section{Personal Response}

Do you think there are other, as yet unidentified, factors which link pacifier use and reduced risk of SIDS; for example, social or environmental influences? II The studies demonstrate a trend towards increas protection when pacifiers are used in an adverse position, sleeping with a mother who smoked, or sleeping on soft bedding. A recent study that sampled found that $73 \%$ of mothers had received no advice from a physician related to pacifier use during sleep. 